\section{Aortic stenosis: flow matters}

\author{
Philippe Pibarot
}

The presence of a low transvalvular pressure gradient $(<40 \mathrm{~mm} \mathrm{Hg})$ in conjunction with a small aortic valve area (AVA $\leq 1 \mathrm{~cm}^{2}$ ) is a challenging situation as it raises uncertainty about the actual severity of aortic stenosis (AS) and therefore about the indication of aortic valve replacement (AVR) if the patient is symptomatic. This lowgradient 'severe' (small AVA) AS entity may in fact be related to: (i) measurement errors: underestimation of stroke volume (SV), AVA and/or gradient; ${ }^{\text {S1 }}$ (ii) small body size: a small AVA in a small patient may correspond to moderate AS and low gradient; (iii) inherent discrepancies in the AVA $\left(\leq 1 \mathrm{~cm}^{2}\right)$ gradient $(\geq 40 \mathrm{~mm} \mathrm{Hg})$ cut-off points proposed in the guidelines ${ }^{\mathrm{S} 2}$ S3 to define severe AS; and (iv) a low-flow state. $^{\mathrm{S} 4}$ Among these four potential causes of low-gradient AS, only the last, that is, the low-flow state, would a priori have a negative impact on outcomes. It is well known that in patients with depressed LV systolic function (LVEF <50\%), the SV and thus the transvalvular flow are often reduced. And in such conditions, the gradient, which is highly flow-dependent, may be low $(<40 \mathrm{~mm} \mathrm{Hg})$ despite the presence of a severe stenosis. ${ }^{\mathrm{S} 4}$ Recent studies and guidelines have also emphasised that LV outflow is often reduced in patients with preserved LVEF and this entity has been named 'paradoxical' low flow. ${ }^{1}$ S2-S4 In these patients, the reduction of $\mathrm{SV}$ is related to pronounced concentric LV remodelling with small LV cavity, impaired diastolic filling and depressed LV longitudinal systolic function (although LVEF is still preserved). ${ }^{1-3}$ S4 S5 Furthermore, other factors frequently encountered in the elderly population with AS may contribute to the low flow including reduced arterial compliance, atrial fibrillation, mitral stenosis, mitral regurgitation and tricuspid regurgitation. ${ }^{2}$

In their Heart paper, Eleid et $a l^{4}$ present the results of an elegant study in which they examined the prognostic value of LV outflow, as expressed by SV index (SVI), in patients with low-gradient AS and preserved LVEF. They showed that lower SVI is incrementally associated with mortality and that the risk of mortality

\footnotetext{
Correspondence to Dr Philippe Pibarot, Department of Medicine, Institut Universitaire de Cardiologie et de Pneumologie de Québec, Laval University, 2725 Chemin Sainte-Foy, Québec, Quebec, Canada G1V-4G5; philippe.pibarot@med.ulaval.ca
}

increases sharply when the SVI becomes lower than $35 \mathrm{~mL} / \mathrm{m}^{2}$.

\section{LOW-GRADIENT AS: WHY IS IT IMPORTANT TO LOOK AT THE FLOW?}

The primary function of the heart is to pump blood. Hence, it would seem logical to use the SV as the primary parameter to assess the heart function. Furthermore, the SV should be indexed for the patient's body surface area to account for inter-individual variation in body size and thus normal cardiac output requirements. Although the $\mathrm{SV}$ is systematically measured in the echocardiography or catheterisation laboratory to calculate cardiac output and AVA, this parameter is generally not used for diagnostic or prognostic purposes. The article of Eleid et $a l^{4}$ provide strong support to the systematic integration of the SVI in the risk stratification and therapeutic management of patients with AS. Although a significant impact on mortality was observed at SVI $<43 \mathrm{~mL} / \mathrm{m}^{2}$, the risk of mortality associated with low flow increased markedly when SVI became $<35 \mathrm{~mL} / \mathrm{m}^{2}$ as shown in the figure 2 of their article. These findings further validate the cut-off value of $35 \mathrm{~mL} / \mathrm{m}^{2}$ proposed by Hachicha et $a l^{1}$ and recently incorporated in the guidelines ${ }^{\mathrm{S} 2} \mathrm{~S} 3$ to define low flow and predict adverse outcomes in AS. However, the study of Eleid et $a l^{4}$ also shows that the mortality rate increases continuously with decreasing SVI and therefore a dichotomisation according to a SVI of $35 \mathrm{~mL} / \mathrm{m}^{2}$ may underestimate the risk of mortality, particularly for patients with SVI between 35 and $43 \mathrm{~mL} / \mathrm{m}^{2}$.

The echocardiographic parameter that is used to measure LV outflow and identify the presence of low flow, that is, the SVI, also has several pitfalls: (i) It is subject to measurements errors, the most frequent being the underestimation of the LV outflow tract diameter that is included in its calculation. $^{\mathrm{S} 1 \mathrm{~S} 4}$ (ii) Obesity may confound the relation between SVI and mortality. Indeed, the indexation of the SV to the patient's body surface area may yield an overestimation of the prevalence and severity of flow low in obese individuals. Furthermore, as reported in the study of Eleid et $a l,{ }^{4}$ obesity is often associated with better survival in the elderly patients with severe AS (ie, the obesity paradox). In their study, Eleid et $a l^{4}$ have paid attention to adjusting for body mass index in the multivariable analysis of the predictors of mortality. In this analysis, body mass index was independently associated with better survival and the association between lower SVI and mortality was even slightly stronger than in univariable analysis.

In summary, the findings of the study of Eleid $e t ~ a l^{4}$ as well as those of previous studies $^{1-3} 5-7$ underline the importance of systematically considering both the gradient and the flow data in the risk stratification and therapeutic management of patients with AS. The gradient is directly related to stenosis severity but inversely related to flow. So a low gradient may be a marker for a less severe stenosis and/or for a low-flow state, and in the latter situation, the gradient may grossly underestimate stenosis severity. Hence, the patients with small AVA and preserved LVEF should be dichotomised according to the mean gradient (high vs low; ie, $\geq$ vs $<40 \mathrm{~mm} \mathrm{Hg}$ ) and the flow (normal vs low; ie, SVI $>$ vs $\leq 35 \mathrm{~mL} / \mathrm{m}^{2}$ ).

\section{MANAGEMENT OF LOW-GRADIENT 'SEVERE' AS}

According to the most recent guidelines, ${ }^{\text {S2 }}$ S3 AVR is reasonable (Class IIa) in symptomatic patients who have low-flow, low-gradient severe AS, who are normotensive, and have an LVEF $\geq 50 \%$ if clinical, haemodynamic and anatomic data support severe valve obstruction as the most likely cause of symptoms. Figure 1 presents a 5-step algorithm to guide the management of patients with low-gradient 'severe' (ie, small AVA) AS and preserved LVEF. The first step should be to rule out potential errors in the measurement of SV, AVA, and mean gradient by using other corroborating methods. The second step is to determine whether or not the patient has low flow because it has important prognostic implications. The study of Eleid et $a l^{4}$ indeed confirms that patients with SVI $<35 \mathrm{~mL} / \mathrm{m}^{2}$ have markedly worse outcomes and should thus receive particular attention. $^{1-3}$ 5-7 S6 S7 On the other hand, patients with low gradient but normal flow generally have a good prognosis ${ }^{2}{ }^{6}$ and can be managed with conservative management and close follow-up, unless they are symptomatic (see Step \#3). The third step is to determine whether or not the patient is symptomatic. If the patient is truly asymptomatic, which can be confirmed with exercise testing, the patient should be managed conservatively and have a close follow-up. If the patient is symptomatic, the fourth step is to determine whether or not the patient is hypertensive. Hypertension is indeed frequent in patients with AS and may contribute to the reduced flow (and thus to the low gradient), symptoms and 
Figure 1 Algorithm for the management of patients with low-gradient AS. The yellow boxes indicate the step of the algorithms boxes, the possible answers to these questions and the outputs of the steps; the green boxes, the disease entity; and the red boxes, the follow-up or therapeutic interventions. AS, aortic stenosis; AVA, aortic valve area; AoV, aortic valve; AVAl, aortic valve area index; AVR, aortic valve replacement; SV, stroke volume; SVI, stroke volume index; MDCT, multi-detector computed tomography; MG, mean gradient. with the questions to address; the blue

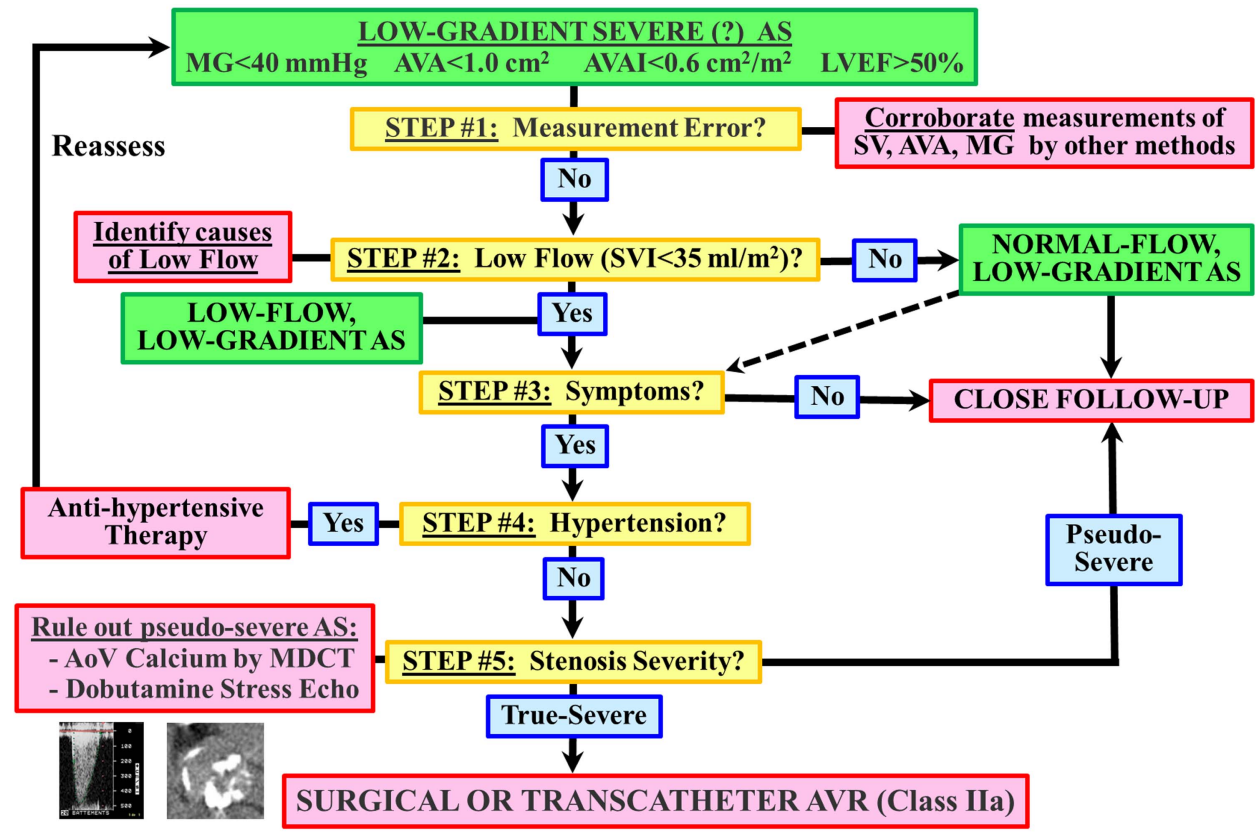

worse outcomes. $^{12}$ If the patient is hypertensive, antihypertensive therapy should be instituted or optimised and the clinical and echocardiographic data should be reassessed after normalisation of blood pressure. ${ }^{\mathrm{S} 3}$ Finally, the last step, but not the least, is to confirm the presence of severe stenosis. Indeed, the presence of low flow makes more complex the assessment of stenosis severity as $30 \%-40 \%$ of the patients with low-flow, low-gradient AS may have pseudo-severe stenosis. ${ }^{8}$ S8 Other diagnostic tests, such as a low dose dobutamine stress echocardiography, may be used to differentiate true versus pseudo severe stenosis. ${ }^{\mathrm{S}}$ However, this test is often not feasible or conclusive in patients with low-flow, low-gradient AS and preserved LVEF, particularly if they have a restrictive physiology pattern. Quantitation of aortic valve calcium load by multi-detector computed tomography (MDCT) may be useful to corroborate stenosis severity in patients with low-gradient AS, independently of the flow or LVEF. However, the cut-off points of aortic valve calcium score that should be used to identify severe stenosis are different in women (>1200 AU) versus men (>2000 AU). ${ }^{8}$ Symptomatic normotensive patients with low-flow, low-gradient AS and evidence of true-severe AS at dobutamine stress echocardiography and/or MDCT should undergo surgical or transcatheter AVR whereas other patients should rather be managed conservatively with optimisation of medical therapy and close clinical and echocardiographic follow-up.

\section{CONCLUSIONS}

This article by Eleid et $a l^{4}$ further emphasises the importance of always interpreting the data of AVA and gradient in light of the flow data. Hence, the SVI should be systematically incorporated in the echocardiographic evaluation and risk stratification of patients with AS and a $\mathrm{SVI}<35 \mathrm{~mL} / \mathrm{m}^{2}$ indicates that the patient is at a higher risk for mortality. Symptomatic patients with paradoxical low-flow, lowgradient AS should undergo further investigations to confirm the presence of severe AS and thus the indication of AVR.

Funding Dr Pibarot holds the Canada Research Chair in Valvular Heart Disease and his research program is funded by the Canadian Institutes of Health research (Ottawa, Ontario, Canada) and the Heart and Stroke Foundation of Québec (Montreal, Québec, Canada).

Competing interests None.

Provenance and peer review Commissioned; internally peer reviewed.

- Additional material is published online only. To view please visit the journal online (http://dx.doi.org/10. 1136/heartjnl-2014-306677).

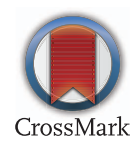

To cite Pibarot P. Heart 2015;101:5-6.

Published Online First 10 November 2014

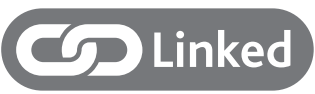

- http://dx.doi.org/10.1136/heartjnl-2014-306151
Heart 2015;101:5-6.

\section{REFERENCES}

1 Hachicha Z, Dumesnil JG, Bogaty P, et al. Paradoxical low flow, low gradient severe aortic stenosis despite preserved ejection fraction is associated with higher 2856-64.

2 Eleid MF, Sorajja P, Michelena HI, et al. Flow-gradient patterns in severe aortic stenosis with preserved ejection fraction: clinical characteristics and predictors of survival. Circulation 2013;128: 1781-9.

3 Mehrotra P, Jansen K, Flynn AW, et al. Differential left ventricular remodelling and longitudinal function distinguishes low flow from normal-flow preserved ejection fraction low-gradient severe aortic stenosis. Eur Heart J 2013;34:1906-14.

4 Eleid M, Sorajja P, Michelena $H$, et al. Survival by stroke volume index in patients with low-gradient normal EF severe aortic stenosis. Heart 2015:101; 23-9.

5 Dumesnil JG, Pibarot P, Carabello B. Paradoxical low flow and/or low gradient severe aortic stenosis despite preserved left ventricular ejection fraction: implications for diagnosis and treatment. Eur Heart J 2010; 31:281-9.

6 Lancellotti P, Magne J, Donal E, et al. Clinical outcome in asymptomatic severe aortic stenosis. Insights from the new proposed aortic stenosis grading classification. J Am Coll Cardiol 2012;59:235-43.

7 Clavel MA, Dumesnil JG, Capoulade R, et al. Outcome of patients with aortic stenosis, small valve area and low-flow, low-gradient despite preserved left ventricular ejection fraction. J Am Coll Cardiol 2012;60:1259-67.

8 Clavel MA, Messika-Zeitoun D, Pibarot P, et al. The complex nature of discordant severe calcified aortic valve disease grading: New insights from combined doppler-echocardiographic and computed tomographic study. I Am Coll Cardiol 2013;62:2329-38. doi:10.1136/heartjnl-2014-306677 afterload and reduced survival. Circulation 2007;115: 